\title{
Desenvolvimento e avaliação da eficiência da estabilização protetora na odontopediatria: um estudo piloto
}

\author{
Development and assessment of the effectiveness of protective \\ stabilization in pediatric dentistry: a pilot study
}

\author{
Rabbith Ive Carolina Moreira Shitsuka* \\ Caleb Shitsuka* \\ Caroline Moraes Moriyama*** \\ Fernanda Nahás Pires Corrêa ${ }^{* * *}$ \\ Carina Sincler Delfino ${ }^{* * * *}$ \\ Maria Salete Nahás Pires Corrêa ${ }^{* * * * *}$
}

\begin{abstract}
Resumo
Objetivo: desenvolver um modelo de estabilização protetora para crianças, com a finalidade de promover qualidade no atendimento e avaliar a sua eficiência na prática clínica. Materiais e método: uma amostra de conveniência composta por vinte cirurgiões dentistas, que atuam no atendimento de crianças com perfil de não colaboradoras. Foi aplicado um questionário com perguntas relacionadas ao conhecimento prévio e à percepção sobre os métodos de estabilização protetora. Com base nas informações coletadas considerando a opinião desses profissionais, foi desenvolvido um novo modelo de estabilização protetora, baseado em modelo já existente (Pedi-Wrap), porém modificado com um tecido mais macio com desenhos para maior conforto e aceitação das crianças e responsáveis. Para composição das peças, os materiais empregados foram compostos por tecidos de algodão e tela garça, velcro, elástico, botões, linhas e viés. O modelo desenvolvido
\end{abstract}

na pesquisa foi utilizado em crianças não colaboradoras de 1 a 6 anos de idade, testados por cada um dos vinte cirurgiões dentistas, em seguida foi aplicado um novo questionário aos profissionais, para se saber a opinião desse novo modelo. Resultados: na opinião dos profissionais, a região de maior movimentação durante um atendimento odontológico é a cabeça das crianças (35\%), o tipo de estabilização mais utilizado é a mãe segurando a criança (35\%), sendo que $45 \%$ dos profissionais avaliados não estão satisfeitos com a estabilização protetora utilizadas no dia a dia. Em relação ao modelo desenvolvido, todos os dentistas acharam eficiente e visualmente agradável. Conclusões: a roupa de estabilização protetora desenvolvida apresentou características como a facilidade na manipulação, agradabilidade e contribuiu no atendimento às crianças.

Palavras-chave: Manejo. Restrição física. Odontopediatria.

Mestre em Odontopediatria, Faculdade de Odontologia da Universidade Cruzeiro do Sul, Departamento de Odontopediatria, São Paulo, SP, Brasil. Mestre em Odontopediatria e doutorando em Ciências Odontológicas, Faculdade de Odontologia da Universidade de São Paulo, Departamento de Ortodontia e Odontopediatria, São Paulo, SP, Brasil.

Mestre em Odontopediatria e doutorando em Ciências Odontológicas, Faculdade de Odontologia da Universidade de São Paulo, Departamento de Ortodontia e Odontopediatria, São Paulo, SP, Brasil.

**** Mestre e Doutora em Odontopediatria, Departamento de Ortodontia e Odontopediatria, São Paulo, SP, Brasil.

Mestre e Doutora em Odontopediatria, Professora Adjunta da Faculdade de Odontologia da Universidade Cruzeiro do Sul, Departamento de Odontopediatria, São Paulo, SP, Brasil.

****** Mestre e Doutora em Odontopediatria, Professora Livre Docente da Faculdade de Odontologia da Universidade de São Paulo, Departamento de Ortodontia e Odontopediatria, São Paulo, SP, Brasil. 


\section{Introdução}

Um grande desafio dos odontopediatras é saber lidar com os sentimentos de medo e ansiedade, frequentemente apresentados pelas crianças no ambiente odontológico ${ }^{1}$. O atendimento deve ser baseado nas técnicas não farmacológicas para administração do comportamento e condicionamento da criança $^{2-4}$.

Um problema comum ocorre quando as crianças não aderem à gestão de comportamento e condicionamento, dificultando o tratamento clínico $^{5-8}$. Assim, o uso de estabilização protetora tem sido aceito como justificativa para o atendimento dessas crianças não colaboradoras ${ }^{9}$.

A definição de estabilização protetora também conhecida como restrição/contenção física é o ato de restringir a liberdade dos movimentos do paciente, para minimizar os riscos de injúrias e danos, permitindo dessa forma a realização do tratamento odontopediátrico com proteção e segurança ${ }^{9,10}$. Entretanto, existem poucas pesquisas acerca dessa temática, pois há desconhecimento e receio por parte dos profissionais e responsáveis na utilização dessa técnica ${ }^{11}$.

Diante disso, o objetivo do estudo foi desenvolver um modelo de estabilização protetora na odontopediatria baseado na experiência prévia e na percepção de cirurgiões dentistas que atuam com crianças não colaboradoras, bem como a avaliação da sua eficiência na prática clínica odontológica.

\section{Materiais e método}

Este estudo foi aprovado pelo Comitê de Ética em Pesquisa da Universidade Cruzeiro do Sul com o protocolo 130/2011. Para o presente estudo, uma amostra de conveniência foi selecionada, composta por vinte cirurgiões-dentistas do curso de especialização em odontopediatria dos centros de ensino Fundecto (Fundação para o Desenvolvimento Científico e Tecnológico da Odontologia) e ABENO (Associação Brasileira de Ensino Odontológico). Durante o curso de especialização, esses profissionais atendem crianças indicadas por outros centros, pelo alto grau de complexidade do tratamento e pela dificuldade do manejo dessas. Então esses são profissionais que frequentemente atendem crianças não colaboradoras.

Desenvolveu-se um questionário como instrumento de coleta de dados, com questões relacionadas aos métodos de estabilização protetora, empregados no atendimento odontológico de crianças não colaboradoras, para se saber o conhecimento prévio e a percepção desses profissionais a respeito do tema, com questões estruturadas e relacionadas aos tipos de estabilização protetora utilizada pelos profissionais, satisfação na sua utilização, pontos que deveriam ser modificados para um melhor atendi- mento e regiões de maior mobilidade da criança durante o tratamento.

Após a amostra selecionada ser orientada sobre o objetivo da pesquisa, (todos eles) assinaram o termo de consentimento e, em seguida, o questionário desenvolvido foi aplicado.

Com base nas informações coletadas sobre a opinião dos profissionais, relacionadas à estabilização protetora da criança no atendimento odontológico, desenvolveu-se uma roupa de estabilização protetora (Figura 1), baseada inicialmente no pedi-wrap, porém modificada, com um tecido mais macio, flexível e com desenhos coloridos. Para composição das peças os materiais empregados foram compostos por tecidos de algodão e tela garça, velcro, elástico, botões, linhas e viés.

\section{FICHA TÉCNICA \\ MODELO: Estabilização protetora \\ DESENHO TÉCNICO
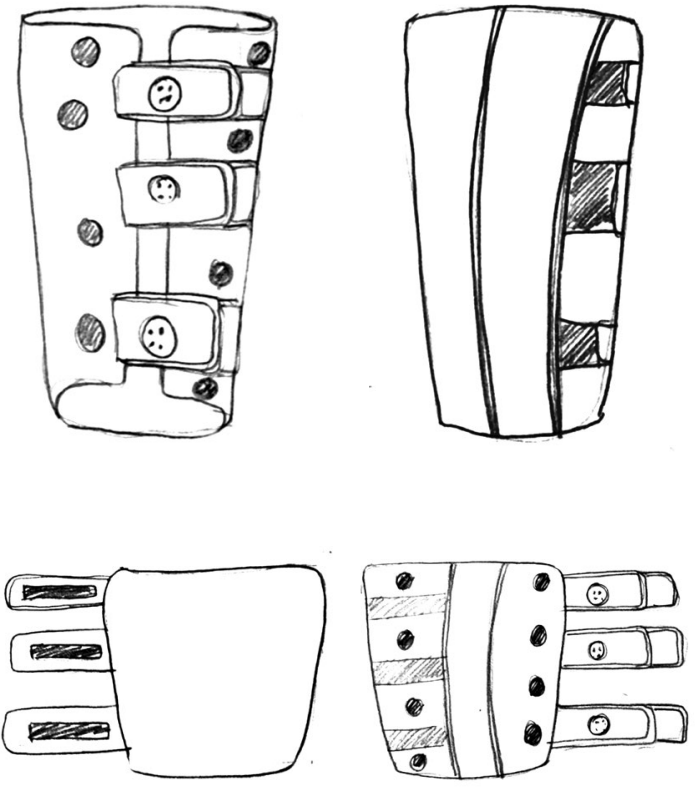 \\ DESCRIÇÃO TECIDOS: \\ - Base: Telagarsa branca com trama pequena \\ - Forro: Forro de algodão branco \\ - Feixes: Existem 3 feixes para abrir e fechar a peça feito com tecido bacterecida \\ - Estampa: Bolinhas laranja feitas com tecido importado, estampa de bolas}

\section{DESCRIÇÃO AVIAMENTOS:}

- Botão: $5 \times 5$ - 015 nas cores azul, vermelho e verde

- Velcro: $5 \mathrm{~cm}$ de largura - 018 na cor branca

- Viés: 2,5 cm - 020 nas cores azul e vermelho

Figura 1 - Modelo da estabilização protetora desenvolvida 
Após a confecção da roupa de estabilização protetora, cada um dos vinte cirurgiões-dentistas testaram-na (Figura 2), utilizando-as no atendimento de vinte crianças de 1 a 6 anos de idade, não colaborativas. Foi aplicado um novo questionário para os profissionais com questões relacionadas à eficiência da estabilização protetora desenvolvida e testada.

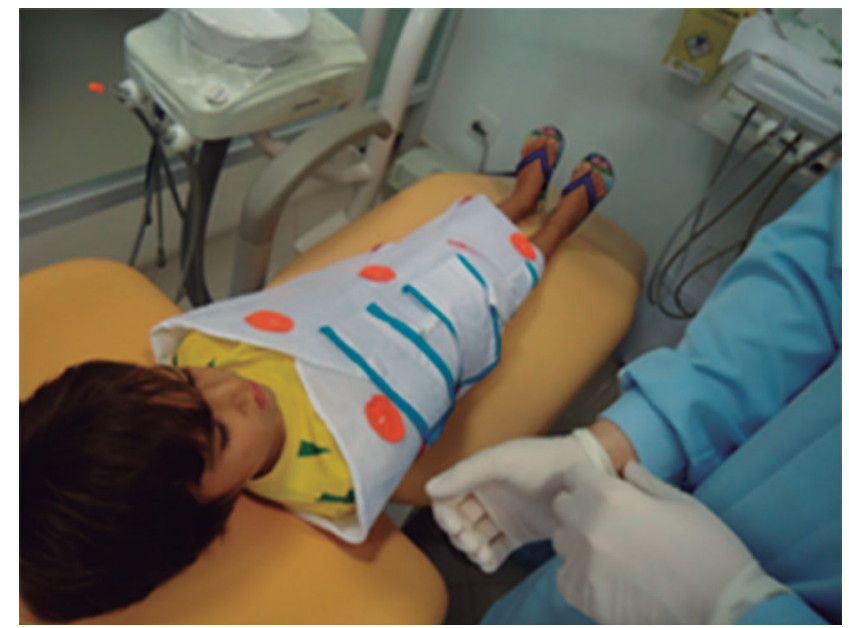

Figura 2 - Utilização da roupa de estabilização protetora

Os resultados obtidos foram analisados estatisticamente e demonstrados em tabelas por porcentagens. No presente estudo, aplicou-se estatística descritiva por meio do Software Minitab versão 14.

\section{Resultados}

Vinte cirurgiões-dentistas participaram deste estudo, todos do sexo feminino, com média de idade ( \pm desvio padrão) de 31.45 (5.13).

Para $35 \%$ dos dentistas, a região de maior mobilidade durante o atendimento odontopediátrico, é a cabeça, seguida dos braços e mãos (Tabela 1).

Tabela 1 - Percepção do dentista sobre a região de maior movimentação das crianças durante o tratamento

\begin{tabular}{l|r}
\hline \multicolumn{1}{c|}{ Região de maior movimentação } & \multicolumn{1}{c}{ n (\%) } \\
\hline Cabeça & $7(35.0)$ \\
Pernas e Pés & $4(20.0)$ \\
Peito e Ombros & $3(15.0)$ \\
Braços e Mãos & $5(25.0)$ \\
Joelhos & $1(5.0)$ \\
Total & $20(100.0)$ \\
\hline
\end{tabular}

As estabilizações protetoras mais utilizadas nos consultórios odontológicos para o atendimento de crianças foram a mãe segurando a criança (35\%) e o pacote pediátrico (30\%). A Tabela 2 mostra que $45 \%$ dos cirurgiões-dentistas não estão satisfeitos com a estabilização protetora utilizadas no atendimento de crianças.
Tabela 2 - Tipos de estabilizações protetoras utilizados pelos dentistas e sua satisfação.

\begin{tabular}{l|r|r|r}
\hline \multirow{2}{*}{$\begin{array}{c}\text { Tipos de } \\
\text { estabilizações }\end{array}$} & $\begin{array}{c}\text { Mais } \\
\text { utilizada } \\
\mathrm{n}(\%)\end{array}$ & $\begin{array}{c}\text { Sim } \mathrm{n} \\
(\%)\end{array}$ & $\begin{array}{c}\text { Não } \mathrm{n} \\
(\%)\end{array}$ \\
\cline { 3 - 4 } & $6(30.0)$ & $3(50.0)$ & $3(50.0)$ \\
Pacote & $1(5.0)$ & $0(0.0)$ & $1(100.0)$ \\
Braços e Pernas & $0(0.0)$ & $0(0.0)$ & $0(0.0)$ \\
Papoose Board & $2(10.0)$ & $1(50.0)$ & $1(50.0)$ \\
Pedi-Wrap & $2(10.0)$ & $2(100.0)$ & $0(0.0)$ \\
Joelho-Joelho & $7(35.0)$ & $3(42.9)$ & $4(57.1)$ \\
Mãe Segurando & $2(10.0)$ & $2(100.0)$ & $0(0.0)$ \\
Auxiliar Segurando & $0(0.0)$ & $0(0.0)$ & $0(0.0)$ \\
Macri & $20(100.0)$ & $11(55.0)$ & $9(45.0)$ \\
\hline Total &
\end{tabular}

A aparência menos agressiva das estabilizações protetoras e a firmeza são os principais pontos que deveriam ser melhorados nas estabilizações protetoras utilizadas (Tabela 3).

Tabela 3 - Percepção dos dentistas sobre quais pontos devem ser meIhorados nas estabilizações protetoras que utilizam

\begin{tabular}{l|r}
\hline \multicolumn{1}{c|}{ O que pode ser aperfeiçoado } & \multicolumn{1}{c}{$\mathrm{n}(\%)$} \\
\hline Firmeza & $8(40.0)$ \\
Aparência menos agressiva & $10(50.0)$ \\
Roupas Confortáveis & $1(5.0)$ \\
Questões Ambientais & $1(5.0)$ \\
\hline
\end{tabular}

Todos os dentistas que testaram a estabilização protetora desenvolvida acharam eficiente e de fácil manipulação, $10 \%$ acharam que ela não conteve os membros superiores e apenas $5 \%$ não utilizaram no cotidiano (Tabela 4).

Tabela 4 - Percepção dos dentistas após a utilização da roupa de estabilização protetora desenvolvida.

\begin{tabular}{l|r|r|r}
\multicolumn{1}{c|}{ Percepção } & $\begin{array}{r}\text { N } \\
\text { Total }\end{array}$ & \multicolumn{1}{c|}{$\begin{array}{c}\text { Sim } \\
\mathrm{n}(\%)\end{array}$} & $\begin{array}{c}\text { Não } \\
\mathrm{n}(\%)\end{array}$ \\
\hline Eficiente & 20 & $20(100.0)$ & $0(0.0)$ \\
Visualmente agradável & 20 & $20(100.0)$ & $0(0.0)$ \\
$\begin{array}{l}\text { Benefício no } \\
\text { atendimento }\end{array}$ & 20 & $20(100.0)$ & $0(0.0)$ \\
$\begin{array}{l}\text { Fácil manipulação } \\
\text { Utilizaria no cotidiano } \\
\text { Conteve membros }\end{array}$ & 20 & $20(100.0)$ & $0(0.0)$ \\
$\begin{array}{l}\text { superiores } \\
\text { Conteve membros }\end{array}$ & 20 & $18(90.0)$ & $2(10.0)$ \\
inferiores & 20 & $13(65.0)$ & $7(35.0)$ \\
\hline
\end{tabular}

\section{Discussão}

A estabilização protetora tem a finalidade de promover qualidade, segurança e evitar injúrias durante o tratamento odontológico, apresentando-se 
como uma alternativa viável aos pacientes infantis que apresentam comportamento não colaborativo ${ }^{12}$.

Diante de uma situação de medo e estresse, a criança pode impedir uma performance adequada do profissional, por meio de movimentos bruscos e repetitivos com o intuito de fuga e auto proteção ${ }^{1}$. Em nosso estudo, a região de maior movimentação na opinião dos dentistas entrevistados, foi à cabeça (35\%). Além da estabilização protetora, a estabilização da cabeça da criança juntamente com o posicionamento do abridor de boca e sugador deve ser feito em equipe, para isso, a auxiliar em saúde bucal é fundamental, pois essa auxilia na redução da ansiedade e no sucesso do tratamento da criança ${ }^{13}$. Contudo, a contenção da região de cabeça e do pescoço deve ser realizada com cuidado, de forma que não ocorra uma pressão na região da artéria carótida, o que traria danos à integridade física do paciente ${ }^{14}$.

É importante enfatizar que a estabilização protetora pode ser utilizada como medida terapêutica, porém com prudência para evitar a sua banalização, pois se utilizada sem corretas indicações, pode acarretar em danos físicos e psíquicos aos pacientes ${ }^{15}$.

A técnica de manejo infantil em que a mãe segura à criança foi a mais frequentemente utilizada no dia a dia clínico dos odontopediatras. Essa aproximação é associada como um fator de ligação, que diminui a ansiedade de ambos e, desse modo, facilita o uso da estabilização protetora ${ }^{16,17}$. Entretanto, dos profissionais que relataram utilizar esse tipo de estabilização protetora, 57\% alegaram insatisfação com essa técnica, isso pode ser explicado pelo fato de que as técnicas menos aceitáveis pelos responsáveis são as de restrição de movimento e de uso de medicamentos ${ }^{18}$, de forma que o responsável pode transmitir um comportamento negativo ao filho durante o tratamento ${ }^{19}$.

Quando não colaboradoras ao tratamento odontológico ${ }^{7}$, apresentando agressividade e excesso de movimentação, dificultando o atendimento, deve haver estabilizações protetoras mais firmes ${ }^{20}$, fato que também foi relatado por $40 \%$ dos odontopediatras.

O principal ponto que os dentistas relataram que deveria ser melhorado é a "aparência menos agressiva" nas estabilizações protetoras já utilizadas. É sabido que as roupas de contenção agregam técnicas para restringir movimentos por meio de algemas, amarras de algodão ou couro ${ }^{21}$, tornando-as visualmente agressivas tanto para o paciente quanto para a equipe profissional. Frente a essas características, deve haver, durante a sessão atributos alegres para facilitar o aspecto psicológico.

Em relação à roupa de contenção desenvolvida, baseada no conhecimento prévio e na percepção dos cirurgiões dentistas, $100 \%$ deles expuseram que a estabilização protetora foi eficiente, visualmente agradável e de fácil manipulação. Esses são itens importantes para serem avaliados, uma vez que a eficiência e a facilidade de manipulação da peça são de suma importância, pois torna a execução do procedimento, controlada e de rápida aplicação ${ }^{17}$.

Apesar da necessidade e da importância da utilização da estabilização protetora no atendimento odontopediatriaco, verificou-se que há escassez na literatura no que diz respeito à utilização desses métodos, o que dificulta a comparação e a busca de relações entre metodologias ou resultados. Diante dessas adversidades, reforça-se a necessidade da realização de pesquisas sobre esse importante assunto da prática clínica dos odontopediatras.

\section{Conclusão}

Apesar da variedade de estabilizações protetoras, $45 \%$ dos dentistas não estavam satisfeitos com a eficiência dessas utilizadas no atendimento. Todos os cirurgiões-dentistas participantes da pesquisa consideraram a roupa de estabilização protetora que foi desenvolvida eficiente, agradável, fácil de manipular e com condições adequadas para o atendimento odontopediatrico.

\section{Abstract}

Objective: to develop a model of protective stabilization for children, in order to promote quality in services and assess its effectiveness in clinical practice. Materials and method: a convenience sample of 20 dentists working with uncooperative children. A questionnaire was applied, with questions related to previous knowledge and perception about the methods of protective stabilization. Based on the information collected and considering the opinion of these professionals, a new model of protective stabilization was developed based on an existing model (Pedi-Wrap), but modified with a softer cloth and pictures, providing greater comfort and acceptance of children and guardians. For the creation of parts, the materials applied were made of cotton and scrim fabric, Velcro, elastic band, buttons, threads, and gore fabric. The model developed in the study was used with uncooperative children from 1 to 6 years old, and tested by each of the 20 dentists. Afterwards, a new questionnaire was applied to the professionals for their opinion on new model. Results: based on the opinion of the professionals, the region with greater movement during dental care is the child's head (35\%) and the most common type of stabilization is to have the mother hold the child (35\%), considering $45 \%$ of professionals assessed were not satisfied with the protective stabilization currently used. Regarding the model developed, all dentists found it effective and visually pleasant. Conclusions: the protective stabilization clothing developed presented features such as easy handling, pleasantness, and it helped during children care.

Keywords: Handling. Physical Restraint. Pediatric Dentistry. 


\section{Referências}

1. Klingberg G, Broberg AG. Dental fear/anxiety and dental behaviour management problems in children and adolescents: a review of prevalence and concomitant psychological factors. Int J Paediatr Dent 2007;17(6):391-406.

2. Adair SM, Schafer TE, Waller JL, Rockman RA. Age and gender differences in the use of behavior management technique by Pediatric Dentist. Pediatr Dent 2007;29(5):403-8.

3. Hoge MA, Howard S, Wallace DP, Allen KD. Use of video eyewear to manage distress in children during restorative dental treatment. Pediatr Dent 2012;34(5)378-53.

4. Carr KR, Wilson S, Nimer S, Thornton JB. Behavior management techniques among pediatric dentists practicing in the southeastern United States. Pediatr Dent 1999;21(6):347-53.

5. Allen KD, Stanley RT, McPherson K. Evaluation of behavior management technology dissemination in pediatric dentistry. Pediatr Dent 1990;12(2):79-82.

6. Ingersoll TG, Ingersoll BD, Seime RS, McCutcheon WR. A survey of patient and auxiliary problems as they relate to behavioral dentistry curricula. J Dent Educ 1978;42:260-63.

7. Suprabha BS, Rao A, Choudhary S, Shenoy R. Child dental fear and behavior: the role of environmental factors in a hospital cohort. J Indian Soc Pedod Prev Dent 2011;29(2):95101.

8. Weinstein P, Nathan JE. The challenge of fearful and phobic children. Dent Clin North Am 1988;32:667-92.

9. Romer M. Consent, restraint, and people with special needs: a review. Spec Care Dentist 2009;29(1):58-66.

10. Connick C, Palat M, Pugliese S. The appropriate use of physical restraint; Considerations. J Dent Child 2000;67:256-62.

11. Folkes K. Is a restraint a form of abuse? Paediatr Nurs 2005;17(6):41-4.

12. American Academy of Pediatric Dentistry. Guideline on behavior guidance for the Pediatric Dental Patient. Pediatr Dent 2006;28(suppl issue):97-105.

13. Zhou Y, Cameron E, Forbes G, Humphris G. Systematic review of the effect of dental staff behaviour on child dental patient anxiety and behaviour. Patient Educ Couns 2011;85(1):4-13.

14. Paterson B, Bradley P, Stark C, Saddler D, Leadbetter D, Allen D. Deaths associated with restraint use in health and social care in the UK. The results of a preliminary survey. Journal of Psychiatric and Mental Health Nursing $2003 ;(10): 3-15$

15. Huizing AR, Hamers JPH, De Jonge J, Candel M, Berger MP. Organisational determinants of the use of physical restraints: a multilevel approach. Soc Sci Med 2007;(65):924-33.

16. Carr EG, Horner RH, Turnbull AP, Marquis JG, McLaughlin DM, McAtee ML, et al. Positive behavior support for people with developmental disabilities: A research synthesis. Washington, D.C.: American Association on Mental Retardation; 1999.

17. Hull K, Clarke D. Are paediatric oncology nurses acknowledging the effects of restraint? A review of the current policy and research. European Journal of Oncology Nursing. 2011;(15):153-518.

18. Murph MG, Machen JB. Parental acceptance of pediatric dentistry behavior management techniques. Pediatr Dent 1984;6(4):193-8.

19. Corkey B, Freeman R. Predictors of dental anxiety in six-year-old children: findings from a pilot study. ASDC J Dent Child 1994;61:267-71.
20. Buchanan H, Niven N. Self-report treatment techniques used by dentists to treat dentally anxious children: a preliminar investigation. Int J Peadiatr Dent 2003;13:9-12.

21. Migon MN, Coutinho ES, Huf G, Adams CE, Cunha GM, Allen MH. Factors associated with the use of physical restraints for agitated patients in psychiatric emergency rooms. Gen Hosp Psychiatry 2008;30(3):263-8.

\section{Endereço para correspondência:}

Caleb Moreira Shitsuka

Faculdade de Odontologia da Universidade de São Paulo Av. Lineu Prestes, 2227

05508-000 São Paulo - SP - Brasil

Telefone: (11) 3091-7835 Fax: (11) 3091-7854

E-mail: caleb@usp.br

Recebido: 16/12/2014. Aceito: 28/04/2015. 